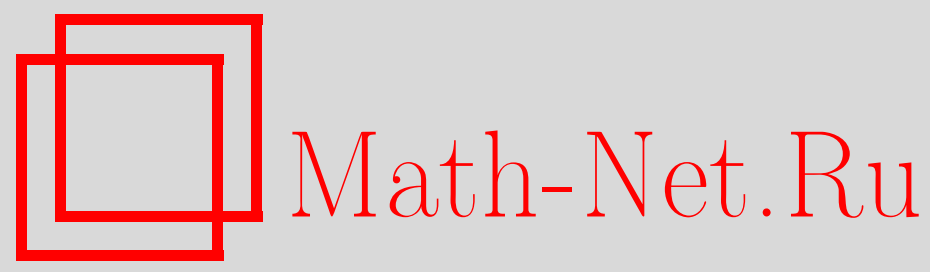

Е. С. Волкова, Тождества кривизны нормальных многообразий киллингова типа, Матем. заметки, 1997, том 62, выпуск 3, 351-362

DOI: https://doi.org/10.4213/mzm1617

Использование Общероссийского математического портала Math-Net.Ru подразумевает, что вы прочитали и согласны с пользовательским соглашением http://www.mathnet.ru/rus/agreement

Параметры загрузки:

IP: 54.84 .234 .179

26 апреля 2023 г., $11: 29: 44$

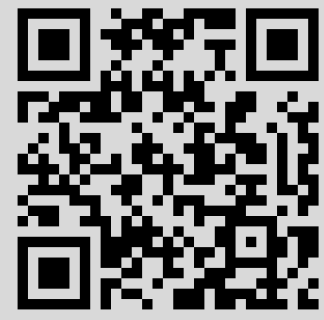




\title{
ТОЖДЕСТВА КРИВИЗНЫ НОРМАЛЬНЫХ МНОГООБРАЗИЙ КИЛЛИНГОВА ТИПА
}

\author{
Е. С. Волкова
}

Вводятся в рассмотрение два тождества кривизны и изучаются соответствующие им классы $R_{1}$ и $R_{2}$ нормальных многообразий киллингова типа.

Библиография: 16 названий.

Геометрия почти контактных метрических многообразий, наделенньх дополнительными структурами, является одним из наиболее содержательных объектов геометрических исследований. Среди таких структур большую роль играют нормальные многообразия киллингова типа (в дальнейшем будем обозначать их CNK-многообразия), обобщающие сасакиевы и квазисасакиевы, косимплектические и слабо косимплектические многообразия, активно изучаемые зарубежными геометрами (Сасаки [1], Исихара [2], Танно [3] и др.). Изучение этого класса многообразий становится более интересным в случае, когда они являются локально конформньпи почти контактньм метрическим многообразиям с замкнутой фундаментальной 2-формой (короче назовем их Сс-многообразия). В этом случае мы получаем контактньй аналог локально конформно-келеровых многообразий, находяших важные приложения в современной математической физике, например, при построении моделей супергравитации в теории Калуцы-Клейна [4].

По выражению А. Грея ключом к изучению геометрии почти эрмитовых структур являются тождества кривизны, выделяющие те или иные классы почти эрмитовых многообразий [5]. Именно на этом пути были выделены такие важные классы почти эрмитовых многообразий, как паракелеровы многообразия, RK-многообразия и т.д. Еще больше тождеств кривизны, выявляющих дополнительные свойства симметрии многообразий, можно выделить для почти контактных многообразий. В настоящей работе вводятся в рассмотрение два тождества кривизны и изучены соответствующие им классы $R_{1}$ и $R_{2}$ почти контактных метрических многообразий. Получены аналитические критерии принадлежности CNK-многообразий к этим классам. Изучено геометрическое строение CNK-многообразий, удовлетворяющих этим тождествам кривизны. Введен оператор $\mathscr{B}$, определяемый тождеством $\mathscr{B}(X)=\nabla_{X} \xi$, где $\xi$ - структурный вектор. Доказано, что распределение $\operatorname{Im} \mathscr{B}$ на $\mathrm{Cc-многообразии} M$ класса $R_{1}$ инволютивно тогда и только тогда, когда $M$ локально эквивалентно произведению квазисасакиева многообразия и келерова многообразия. Получено несколько критериев интегрируемости структуры CNK-многообразия $M$.

Пусть $M^{2 n+1}$ - гладкое многообразие с почти контактной метрической структурой $\{\Phi, \xi, \eta, g=\langle\cdot, \cdot\rangle\}, \eta(\xi)=1, \Phi(\xi)=0, \eta \circ \Phi=0, \Phi^{2}=-\mathrm{id}+\xi \otimes \eta,\langle\Phi X, \Phi Y\rangle=\langle X, Y\rangle-$ 
$\eta(X) \eta(Y), \mathscr{X}(M)$ - алгебра Ли гладких векторных полей на $M, d$ - оператор внешнего дифференцирования, $\nabla$ - риманова связность на $M, \Omega(X, Y)=\langle X, \Phi Y\rangle$ - фундаментальная форма структуры, $C^{\infty}(M)$ - алгебра гладких функций многообразия $M$.

Напомним [6], что почти контактная метрическая структура назьвается нормальной, если $2 N+\xi \otimes d \eta=0$, где

$$
N(X, Y)=\frac{1}{4}\left([\Phi X, \Phi Y]+\Phi^{2}[X, Y]-\Phi[\Phi X, Y]-\Phi[X, \Phi Y]\right)
$$

- тензор Нейенхейса структурного оператора $\Phi$.

Нормальную почти контактную метрическую структуру с киллинговым структурным ковектором назовем CNK-структурой.

Киллинговость структурного ковектора означает, что верно тождество

$$
\nabla_{X}(\eta) Y+\nabla_{Y}(\eta) X=0, \quad X, Y \in \mathscr{X}(M) .
$$

Известно [7], что задание почти контактной метрической структуры на $M^{2 n+1}$ определяет в модуле $\mathscr{X}(M)$ пару взаимно-дополнительных проекторов $m=\xi \otimes \eta, l=\mathrm{id}-\xi \otimes \eta$. Известно также, что $l=-\Phi^{2},\langle\Phi l X, \Phi l Y\rangle=\langle l X, l Y\rangle, X, Y \in \mathscr{X}(M)$. Это означает, что на распределении $\mathscr{L}=\operatorname{Im} \Phi$ пара $\{\Phi|\mathscr{L}, g| \mathscr{L}\}$ определяет почти эрмитову структуру, следовательно, $\mathscr{L}$ можно рассматривать как эрмитово векторное расслоение над $M$ с метрикой $H(X, Y)=\langle X, Y\rangle+\sqrt{-1}\langle X, \Phi Y\rangle, X, Y \in \mathscr{X}(M)$.

Рассмотрим теперь комплексификацию [8] расслоения $\mathscr{L}$ - расслоение $\mathscr{L}^{\mathbb{C}}=\mathscr{L} \otimes \mathbb{C}$ над $M$. В расслоении $\mathscr{L}^{\mathbb{C}}$ естественно определены взаимно-дополнительные проекторы $\sigma=\frac{1}{2}(\mathrm{id}-\sqrt{-1} \Phi \mid \mathscr{L}), \bar{\sigma}=\frac{1}{2}\left(\mathrm{id}+\left.\sqrt{-1} \Phi\right|_{\mathscr{L}}\right)$ (здесь под оператором $\left.\Phi\right|_{\mathscr{L}}$, вообще говоря, понимается его комплексификация $\left.\Phi^{\mathbb{C}}=(\Phi \mid \mathscr{L}) \otimes \mathrm{id}_{\mathbb{C}}\right)$, а также оператор $\tau$ естественного сопряжения, причем, очевидно, что на $\mathscr{L}$ справедливо соотношение $\bar{\sigma}=\tau$ о $\sigma[7]$.

Задание проекторов $\sigma$ и $\bar{\sigma}$ равносильно распадению модуля $\mathscr{L}^{\mathbb{C}}$ в прямую сумму двух подмодулей $D$ и $\bar{D}$ - образов этих проекторов. Легко видеть, что $D$ и $\bar{D}$ являются собственньпи подмодулями оператора $\Phi$ с собственньми значениями $\sqrt{-1}$ и $-\sqrt{-1}$ соответственно. Итак, верно соотношение $\sigma \circ \Phi=\Phi \circ \sigma=\sqrt{-1} \sigma$, т.е. $\Phi(\sigma X)=\sqrt{-1}(\sigma X)$, $X \in \mathscr{L}$. Таким образом, $D=\operatorname{Im} \sigma$ будет собственным подмодулем оператора $\Phi$ с собственньм значением $\sqrt{-1}$. Аналогично, $\bar{\sigma} \circ \Phi=\Phi \circ \bar{\sigma}=-\sqrt{-1} \sigma, X \in \mathscr{L}$, т.е. $\bar{D}=\operatorname{Im} \bar{\sigma}$ будет собственным подмодулем оператора $\Phi$ с собственным значением $-\sqrt{-1}$.

Пусть $p \in M$. Выберем в $\mathscr{L}_{p}$ репер $\left\{p, e_{1}, \ldots, e_{n}\right\}$, унитарньй относительно метрики $H$. Построим репер $\left\{p, E_{o}, E_{1}, \ldots, E_{n}, E_{\widehat{1}}, \ldots, E_{\widehat{n}}\right\}$ модуля $\mathscr{L}_{p}^{\mathbb{C}} \oplus \mathscr{M}_{p}$, где $\mathscr{M}_{p}=$ $\operatorname{Im}\left(m_{p}\right)$, положив $E_{o}=\xi, E_{a}=\sigma\left(e_{a}\right), E_{\widehat{a}}=\bar{\sigma}\left(e_{a}\right)$, где условимся считать, что индексы $i, j, k, s$ пробегают значения от 0 до $2 n$, а индексы $a, b, c, d, g$ - значения от 1 до $n$, $\widehat{a}=a+n$. Заметим, что $\Phi\left(E_{a}\right)=\Phi\left(\sigma\left(e_{a}\right)\right)=\sqrt{-1} E_{a}, \Phi\left(E_{\widehat{a}}\right)=\Phi\left(\bar{\sigma}\left(e_{a}\right)\right)=-\sqrt{-1} E_{\widehat{a}}$, $\Phi\left(E_{o}\right)=\Phi(\xi)=0$.

Репер $\left\{p, E_{o}, E_{1}, \ldots, E_{n}, E_{\widehat{1}}, \ldots, E_{\widehat{n}}\right\}$ называется репером, адаптированным структуре, или $A$-репером [9]. Известно [7], что задание почти контактной метрической структуры на многообразии $M^{2 n+1}$ равносильно заданию $G$-структуры на пространстве расслоения всех реперов на многообразии $M^{2 n+1}$ со структурной групой $G=$ $\mathrm{U}(n) \times\{1\} . A$-реперы характеризуются тем, что матрицы тензоров $\Phi$ и $g$ в них имеют соответственно вид

$$
\left(\Phi_{j}^{i}\right)=\left(\begin{array}{ccc}
0 & 0 & 0 \\
0 & \sqrt{-1} I_{n} & 0 \\
0 & 0 & -\sqrt{-1} I_{n}
\end{array}\right), \quad\left(g_{i j}\right)=\left(\begin{array}{ccc}
1 & 0 & 0 \\
0 & 0 & I_{n} \\
0 & I_{n} & 0
\end{array}\right),
$$


где $I_{n}-$ единичная матрица порядка $n$.

Пусть $\omega$ - форма смещения, $\varphi$ - форма римановой связности $\nabla$ на пространстве присоединенной $G$-структуры. На пространстве этой $G$-структуры форма связности $\varphi$ задается формами $\left\{\omega_{j}^{i}\right\}$, форма смещения $\omega$ - формами $\left\{\omega^{i}\right\}$. Первая группа структурных уравнений почти контактного метрического многообразия имеет вид

$$
\begin{aligned}
1) d \omega^{o}= & -\sqrt{-1} \Phi_{[a, b]}^{o} \omega^{a} \wedge \omega^{b}+\sqrt{-1} \Phi_{[\widehat{a}, \widehat{b}]}^{o} \omega^{\widehat{a}} \wedge \omega^{\widehat{b}}+\sqrt{-1} \Phi_{\widehat{a}, o}^{o} \omega^{\widehat{a}} \wedge \omega^{o} \\
& -\sqrt{-1}\left(\Phi_{a, \widehat{b}}^{o}+\Phi_{\widehat{b}, a}^{o}\right) \omega^{a} \wedge \omega^{\widehat{b}}-\sqrt{-1} \Phi_{a, o}^{o} \omega^{a} \wedge \omega^{o} ; \\
2) d \omega^{a}= & \omega_{b}^{a} \wedge \omega^{b}-\frac{\sqrt{-1}}{2} \Phi_{\widehat{b}, c}^{a} \omega^{c} \wedge \omega^{\widehat{b}}+\frac{\sqrt{-1}}{2} \Phi_{[\widehat{b}, \widehat{c}]}^{a} \omega^{\widehat{b}} \wedge \omega^{\widehat{c}} \\
& -\sqrt{-1} \Phi_{o, b}^{a} \omega^{b} \wedge \omega^{o}+\left(\frac{\sqrt{-1}}{2} \Phi_{\widehat{b}, o}^{a}+\sqrt{-1} \Phi_{o, \widehat{b}}^{a}\right) \omega^{\widehat{b}} \wedge \omega^{o} ;
\end{aligned}
$$

3) $d \omega^{\widehat{a}}=\omega_{\widehat{b}}^{\widehat{a}} \wedge \omega^{\widehat{b}}+\frac{\sqrt{-1}}{2} \Phi_{b, \widehat{c}}^{\widehat{a}} \omega^{\widehat{c}} \wedge \omega^{b}-\frac{\sqrt{-1}}{2} \Phi_{[\widehat{b}, \widehat{c}]}^{\widehat{a}} \omega^{b} \wedge \omega^{c}$

$$
+\sqrt{-1} \Phi_{o, \widehat{b}}^{\widehat{a}} \omega^{\widehat{b}} \wedge \omega_{o}+\left(\frac{\sqrt{-1}}{2} \Phi_{b, o}^{\widehat{a}}-\sqrt{-1} \Phi_{o, b}^{\widehat{a}}\right) \omega^{b} \wedge \omega^{o} .
$$

Эти уравнения были получены В. Ф. Кириченко в работе [7].

Записьвая тождества, характеризующие CNK-структуру на пространстве присоединенной $G$-структуры, получим первую группу структурных уравнений CNK-многообразия:

$$
\begin{gathered}
d \omega=2 B_{a}{ }^{b} \omega^{a} \wedge \omega_{b}, \\
d \omega^{a}=\omega_{b}^{a} \wedge \omega^{b}+B^{a b}{ }_{c} \omega^{c} \wedge \omega_{b}-B_{b}{ }^{a} \omega^{b} \wedge \omega \\
d \omega_{a}=-\omega_{a}^{b} \wedge \omega_{b}+B_{a b}{ }^{c} \omega^{b} \wedge \omega_{c}+B_{a}{ }^{b} \omega_{b} \wedge \omega
\end{gathered}
$$

где

$$
\begin{gathered}
B_{a}^{b}=\sqrt{-1} \Phi_{o, \widehat{b}}^{\widehat{a}}=-\sqrt{-1} \Phi_{a, \widehat{b}}^{o}, \quad B^{a b}{ }_{c}=-\frac{\sqrt{-1}}{2} \Phi_{\widehat{b}, c}^{a}, \\
B_{a b}{ }^{c}=-\frac{\sqrt{-1}}{2} \Phi_{b, \widehat{c}}^{\widehat{a}}, \quad \overline{B_{a}^{b}}=-B_{b}{ }^{a}, \quad \overline{B^{a b}{ }_{c}}=-B_{a b}{ }^{c}
\end{gathered}
$$

(здесь и далее будем считать $\omega^{o}=\omega$ ).

Применяя к этим тождествам стандартную процедуру дифференциального продолжения [7], получим вторую группу структурньх уравнений CNK-многообразия:

$$
\begin{aligned}
& d \omega_{b}^{a}=\omega_{c}^{a} \wedge \omega_{b}^{c}+\left(A_{b d}^{a c}-2 B_{b}{ }^{a} B_{d}^{c}\right) \omega^{d} \wedge \omega_{c}+A_{b c d}^{a} \omega^{c} \wedge \omega^{d}+A_{b}^{a c d} \omega_{c} \wedge \omega_{d} \\
& -\left(C_{b d}^{a}+B_{[b}{ }^{h} B_{d] h}{ }^{a}\right) \omega^{d} \wedge \omega-\left(C_{b}^{a d}-B_{h}{ }^{[a} B^{d] h}{ }_{b}\right) \omega_{d} \wedge \omega, \\
& d B_{b}{ }^{a}=B_{b}{ }^{g} \omega_{g}^{a}-B_{g}{ }^{a} \omega_{b}^{g}+\left(C_{b g}^{a}+B_{[b}{ }^{h} B_{g] h}{ }^{a}\right) \omega^{g}+\left(C_{b}^{a g}-B_{h}{ }^{[a} B^{g] h}{ }_{b}\right) \omega_{g}, \\
& d B_{a b}{ }^{c}=B_{a b}{ }^{g} \omega_{g}^{c}-B_{g b}{ }^{c} \omega_{a}^{g}-B_{a g}{ }^{c} \omega_{b}^{g}+B_{a b}{ }^{c} \omega^{g}+B_{a b} c g \omega_{g} \\
& +\left(B_{a b}{ }^{g} B_{g}{ }^{c}-B_{g b}{ }^{c} B_{a}{ }^{g}-B_{a g}{ }^{c} B_{b}{ }^{g}\right) \omega,
\end{aligned}
$$

где

$$
\begin{gathered}
\overline{A_{c d}^{a b}}=A_{a b}^{c d}, \overline{A_{b c d}^{a}}=-A_{a}^{b c d}, \quad \overline{C_{b c}^{a}}=-C_{a}^{b c}, \\
\overline{B^{a b} c d}=-B_{a b}{ }^{c d}, \overline{B^{a b}{ }_{c}{ }^{d}}=-B_{a b}{ }^{c} d, \quad C_{b c}^{a}=C_{c b}^{a} .
\end{gathered}
$$


Соотношения (2) и (4) представляют собой системы функций на пространстве присоединенной $G$-структуры, служашие компонентами соответствуюших структурных тензоров, а квадратные и круглые скобки означают соответственно операции альтернирования и симметризации объекта по заключенньм в них индексам.

Далее, приведем несколько полезных соотношений, верных для CNK-многообразий:

$$
\begin{gathered}
\omega_{\widehat{b}}^{a}=B^{a b}{ }^{c} \omega^{c}, \quad \omega_{b}^{\widehat{a}}=-B_{a b}{ }^{c} \omega_{c}, \quad \omega_{o}^{a}=-B_{b}{ }^{a} \omega^{b} \\
\omega_{o}^{\widehat{a}}=B_{a}{ }^{b} \omega_{b}, \quad \omega_{a}^{o}=-B_{a}{ }^{b} \omega_{b}, \quad \omega_{\widehat{a}}^{o}=B_{b}{ }^{a} \omega^{b} .
\end{gathered}
$$

Рассматривая второе уравнение Картана связности $\nabla$ [10] и используя соотношения (1), (3) и (5), получаем спектр тензора Римана-Кристоффеля, т.е. выражение его компонент на пространстве присоединенной $G$-структуры:

$$
\begin{gathered}
R_{b c d}^{a}=2 A_{b c d}^{a}, \quad R_{b c \widehat{d}}^{a}=A_{b c}^{a d}-2 B_{b}{ }^{a} B_{c}{ }^{d}-B_{c}{ }^{a} B_{b}{ }^{d}+B^{a h}{ }_{c} B_{h b}{ }^{d}, \\
R_{b c o}^{a}=-C_{b c}^{a}-B_{[b}{ }^{h} B_{c] h}{ }^{a}, \quad R_{\widehat{b} c d}^{a}=2\left(B^{a b}{ }_{[d c]}+B_{[c}{ }^{a} B_{d]}{ }^{b}\right), \quad R^{a}{ }_{o b o}=B_{b}{ }^{h} B_{h}{ }^{a}, \\
R_{\widehat{b} \widehat{c} \widehat{d}}^{a}=R_{\widehat{b} \widehat{c} o}^{a}=0, \quad R_{\widehat{b} c o}^{a}=2 B_{h}{ }^{[a} B^{b] h}{ }_{c}, \quad R^{a}{ }_{o \widehat{b} o}=0 .
\end{gathered}
$$

Остальные компоненты тензора Римана-Кристоффеля можно определить из вьписанных, учитывая свойства симметрии и вещественность тензора кривизны римановой связности.

В геометрии CNK-многообразий особую роль играет оператор $\mathscr{B}(X)=\nabla_{X} \xi$. Вычислим его компоненты на пространстве присоединенной $G$-структуры. Его компонентами $\left\{\xi_{, k}^{i}\right\}$ являются компоненты ковариантной производной структурного вектора. Из тензорного анализа известно [11], что на пространстве рассматриваемых реперов над $M$ выполняются дифференциальные уравнения

$$
d \xi^{i}-\xi^{j} \omega_{j}^{i}=\xi_{, k}^{i} \omega^{k}
$$

в частности, на пространстве присоединенной $G$-структуры будем иметь

$$
d \xi^{a}-\xi^{b} \omega_{b}^{a}-\xi^{\widehat{b}} \omega_{\widehat{b}}^{a}-\xi^{o} \omega_{o}^{a}=\xi_{, k}^{a} \omega^{k}
$$

Принимая во внимание соотношения $\xi^{a}=\xi_{a}=0, \xi^{o}=1$, а также учитьвая соотношение (5) для $\omega_{o}^{a}$, находим

$$
B_{b}{ }^{a} \omega^{b}=\xi_{, b}^{a} \omega^{b}+\xi_{, \widehat{b}}^{a} \omega^{\widehat{b}}+\xi_{, o}^{a} \omega .
$$

Учитьвая линейную независимость базисных форм $\left\{\omega^{a}, \omega_{a}, \omega\right\}$, получим

$$
\xi_{, b}^{a}=B_{b}^{a}, \quad \xi_{, \widehat{b}}^{a}=0, \quad \xi_{, o}^{a}=0
$$

Далее,

$$
d \xi^{\widehat{a}}-\xi^{b} \omega_{b}^{\widehat{a}}-\xi^{\widehat{b}} \omega_{\widehat{b}}^{\widehat{a}}-\xi^{o} \omega_{o}^{\widehat{a}}=\xi_{, k}^{\widehat{a}} \omega^{k}, \quad d \xi^{o}-\xi^{b} \omega_{b}^{o}-\xi^{\widehat{b}} \omega_{\widehat{b}}^{o}-\xi^{o} \omega_{o}^{o}=\xi_{, k}^{o} \omega^{k} .
$$

Аналогично, находим

$$
\xi_{, b}^{\widehat{a}}=0, \quad \xi_{, \widehat{b}}^{\widehat{a}}=-B_{a}^{b}, \quad \xi_{, o}^{\widehat{a}}=0, \quad \xi_{, k}^{o}=0
$$


С другой стороны, из определения оператора $\mathscr{B}$ имеем

$$
\begin{aligned}
& \mathscr{B}_{a}^{b}=\left(\mathscr{B}\left(E_{a}\right)\right)^{b}=\left(\nabla_{E_{a}} \xi\right)^{b}=\xi_{, a}^{b}=B_{a}^{b}, \\
& \mathscr{B}_{a}^{\widehat{b}}=\left(\mathscr{B}\left(E_{a}\right)\right)^{\widehat{b}}=\left(\nabla_{E_{a}} \xi\right)_{, a}^{\widehat{b}}=0, \\
& \mathscr{B}_{a}^{o}=\left(\mathscr{B}\left(E_{a}\right)\right)^{o}=\left(\nabla_{E_{a}} \xi\right)^{o}=\xi_{, a}^{o}=0 .
\end{aligned}
$$

Таким образом, оператор $\mathscr{B}$ переводит распределение $D_{\Phi}^{\sqrt{-1}}$ в себя, а $\left\{B_{b}{ }^{a}\right\}$ - это компоненты сужения оператора $\mathscr{B}$ на $D_{\Phi}^{\sqrt{-1}}$. Итак, матрица оператора $\mathscr{B}$ в $A$-репере имеет вид

$$
(\mathscr{B})_{j}^{i}=\left(\begin{array}{ccc}
0 & 0 & 0 \\
0 & B_{b}{ }^{a} & 0 \\
0 & 0 & -B_{a}{ }^{b}
\end{array}\right) .
$$

Поскольку $\mathscr{B}$ - тензор типа $(1,1)$, для его компонент верно соотношение

$$
d \mathscr{B}_{j}^{i}+\mathscr{B}_{k}^{i} \omega_{j}^{k}-\mathscr{B}_{j}^{k} \omega_{k}^{i}=\mathscr{B}_{i, k}^{i} \omega^{k},
$$

где $\{\mathscr{B}\}_{j, k}^{i}-$ компоненты $\nabla \mathscr{B}$. Отсюда, расписывая его на пространстве присоединенной $G$-структуры с учетом (7), находим

$$
\begin{gathered}
\mathscr{B}_{b, c}^{a}=C_{b c}^{a}+B_{[b}{ }^{h} B_{c] h}{ }^{a}, \quad \mathscr{B}_{\widehat{b}, \widehat{c}}^{\widehat{a}}=-C_{a}^{b c}+B_{h}{ }^{[b} B^{c] h}{ }_{a}, \\
\mathscr{B}_{o, b}^{a}=-B_{c}{ }^{a} B_{b}{ }^{c}, \quad \mathscr{B}_{\widehat{b}, c}^{\widehat{a}}=-C_{a c}^{b}-B_{[a}{ }^{h} B_{c] h}{ }^{b}, \\
\mathscr{B}_{\widehat{a}, b}^{o}=B_{c}{ }^{a} B_{b}{ }^{c}, \quad \mathscr{B}_{b, \widehat{c}}^{a}=C_{b}^{a c}-B_{h}{ }^{[a} B^{c] h}{ }_{b}, \\
\mathscr{B}_{o, \widehat{b}}^{\widehat{a}}=-B_{a}{ }^{c} B_{c}{ }^{b}, \quad \mathscr{B}_{a, \widehat{b}}^{o}=B_{a}{ }^{c} B_{c}{ }^{b}, \quad \mathscr{B}_{\widehat{b}, c}^{a}=2 B_{h}{ }^{b} B^{a] h}{ }_{c}, \quad \mathscr{B}_{b, \widehat{c}}^{\widehat{a}}=2 B_{[b}{ }^{h} B_{a] h}{ }^{b},
\end{gathered}
$$

остальные компоненты тензора $\nabla \mathscr{B}$ равны 0 .

Сравнивая компоненту тензора Римана-Кристоффеля $R_{b o c}^{a}$ из соотношений (6) и компоненту $\mathscr{B}_{b, c}^{a}(8)$ оператора $\nabla \mathscr{B}$, видим, что $R_{b o c}^{a}=\mathscr{B}_{b, c}^{a}$. Кроме того, имеем (см. (6)) $R_{b o c}^{\widehat{a}}=0, R_{b o c}^{o}=0$, а также из соотношений (8) $\mathscr{B}_{b, c}^{\widehat{a}}=\mathscr{B}_{b, c}^{o}=0$. Но $R_{b o c}^{a}=\mathscr{B}_{b, c}^{a}$ тогда и только тогда, когда

$$
\left(R\left(E_{o}, E_{c}\right) E_{b}\right)^{a}=\left(\nabla_{E_{c}}(\mathscr{B}) E_{b}\right)^{a} .
$$

Кроме того,

$$
\left(R\left(E_{o}, E_{c}\right) E_{b}\right)^{\widehat{a}}=\left(\nabla_{E_{c}}(\mathscr{B}) E_{b}\right)^{\widehat{a}}=0, \quad\left(R\left(E_{o}, E_{c}\right) E_{b}\right)^{o}=\left(\nabla_{E_{c}}(\mathscr{B}) E_{b}\right)^{o}=0 .
$$

Учитьвая эти тождества, получим $R\left(E_{o}, E_{c}\right) E_{b}=\nabla_{E_{c}}(\mathscr{B}) E_{b}$. Так как $E_{o}, E_{a}$ - базисные векторы подпространства $\left(\mathscr{M} \oplus D_{\Phi}^{\sqrt{-1}}\right)_{p}, p \in M$, то верно тождество

$$
R(\xi, \bar{X}) \bar{Y}=\nabla_{\bar{X}}(\mathscr{B}) \bar{Y}, \quad \bar{X}, \bar{Y} \in D_{\Phi}^{\sqrt{-1}} .
$$


Значит, вьполняется соотношение

$$
R(\xi, \sigma X) \sigma Y=\nabla_{\sigma X}(\mathscr{B}) \sigma Y, \quad X, Y \in \mathscr{L}^{\mathbb{C}}
$$

где $\sigma=\frac{1}{2}(\mathrm{id}-\sqrt{-1} \Phi)-$ проектор на $\mathscr{L}^{\mathbb{C}}$. Поэтому получаем

$$
R\left(\xi, \frac{X-\sqrt{-1} \Phi X}{2}\right)\left(\frac{Y-\sqrt{-1} \Phi Y}{2}\right)=\nabla_{(X-\sqrt{-1} \Phi X) / 2}(\mathscr{B})\left(\frac{Y-\sqrt{-1} \Phi Y}{2}\right)
$$

Раскроем это выражение по линейности:

$$
\begin{aligned}
& R(\xi, X) Y-\sqrt{-1} R(\xi, \Phi X) Y-\sqrt{-1} R(\xi, X)(\Phi Y)-R(\xi, \Phi X)(\Phi Y) \\
& \quad=\nabla_{X}(\mathscr{B}) Y-\sqrt{-1} \nabla_{\Phi X}(\mathscr{B}) Y-\sqrt{-1} \nabla_{X}(\mathscr{B})(\Phi Y)-\nabla_{\Phi X}(\mathscr{B})(\Phi Y), \quad X, Y \in \mathscr{L}^{\mathbb{C}} .
\end{aligned}
$$

Выделим в последнем выражении действительную и мнимую части:

$$
\begin{aligned}
& R(\xi, X) Y-R(\xi, \Phi X)(\Phi Y)=\nabla_{X}(\mathscr{B}) Y-\nabla_{\Phi X}(\mathscr{B})(\Phi Y) \\
& R(\xi, \Phi X) Y+R(\xi, X)(\Phi Y)=\nabla_{\Phi X}(\mathscr{B}) Y+\nabla_{X}(\mathscr{B})(\Phi Y)
\end{aligned}
$$

Эти равенства эквивалентны, так как меняя в первом из них $X$ на $\Phi X$, а $Y$ на $\Phi Y$ и учитьвая, что $\left(\left.\Phi\right|_{\mathscr{L} \mathbb{C}}\right)^{2}=-\mathrm{id}$, получим второе равенство. Итак, равенство (9) эквивалентно следующему:

$$
R(\xi, X) Y-R(\xi, \Phi X)(\Phi Y)=\nabla_{X}(\mathscr{B}) Y-\nabla_{\Phi X}(\mathscr{B})(\Phi Y), \quad X, Y \in \mathscr{L}^{\mathbb{C}}
$$

Так как проектором на $\mathscr{L}^{\mathbb{C}}$ является эндоморфизм $l=-\Phi^{2}$, то с учетом $\Phi^{3}=-\Phi$, получим

$$
R\left(\xi, \Phi^{2} X\right)\left(\Phi^{2} Y\right)-R(\xi, \Phi X)(\Phi Y)=\nabla_{\Phi X}(\mathscr{B})\left(\Phi^{2} Y\right)-\nabla_{\Phi X}(\mathscr{B})(\Phi Y), \quad X, Y \in \mathscr{X}(M) .
$$

Назовем это тождество первьм тождеством кривизны CNK-многообразия.

ОПРЕДЕЛЕНИЕ 1. СNK-многообразие называется многообразием класса $R_{1}$, если

$$
R\left(\Phi^{2} Y, \xi\right)\left(\Phi^{2} X\right)=R(\Phi Y, \xi)(\Phi X), \quad X, Y \in \mathscr{X}(M)
$$

Из первого тождества кривизны следует, что CNK-многообразие принадлежит классу $R_{1}$ тогда и только тогда, когда

$$
\nabla_{X}(\mathscr{B}) Y=\nabla_{\Phi X}(\mathscr{B})(\Phi Y), \quad X, Y \in \mathscr{X}(M)
$$

Теперь докажем следующее

ПРЕДЛОЖЕНИЕ 1. Пусть $M-\mathrm{CNK}$-многообразие, тогда

$$
\langle\mathscr{B}(X), Y\rangle=-\langle X, \mathscr{B}(Y)\rangle, \quad X, Y \in \mathscr{X}(M)
$$


ДоказАТЕЛЬСтво. Имеем $\langle\mathscr{B}(X), Y\rangle=g_{i j} \mathscr{B}_{k}^{i} X^{k} Y^{j}$. Учитьвая значения компонент оператора $\mathscr{B}(7)$, получаем

$$
\langle\mathscr{B}(X), Y\rangle=B_{c}^{b} X^{c} Y^{\widehat{b}}-B_{b}^{c} X^{\widehat{c}} Y^{b}
$$

Аналогично,

$$
\langle X, \mathscr{B}(Y)\rangle=-B_{c}^{b} X^{c} Y^{\widehat{b}}+B_{b}^{c} X^{\widehat{c}} Y^{b}
$$

Сравнивая эти выражения, получаем $\langle\mathscr{B}(X), Y\rangle=-\langle X, \mathscr{B}(Y)\rangle$.

ПРЕДЛОЖЕНИЕ 2. Пусть $M$ - СNK-многообразие, $\mathscr{B}$ - оператор, определяемый формулой $\mathscr{B}(X)=\nabla_{X} \xi, X \in \mathscr{X}(M)$. Тогда $\mathscr{X}(M)=\operatorname{Im} \mathscr{B} \oplus \operatorname{Ker} \mathscr{B}$.

ДокАЗАтЕльство. 1) Пусть $X \in(\operatorname{Im} \mathscr{B})^{\perp}$. Тогда для всех $Y \in \mathscr{X}(M)$ имеем $\mathscr{B}(Y) \in \operatorname{Im} \mathscr{B}$ и $\langle\mathscr{B}(Y), X\rangle=0$. В силу (10) верно соотношение $\langle\mathscr{B}(X), Y\rangle=0$. Учитывая невырожденность метрики, находим $\mathscr{B}(X)=0$, т.е. $X \in \operatorname{Ker} \mathscr{B}$. Итак, для всех $X \in(\operatorname{Im} \mathscr{B})^{\perp}$ выполнено $X \in \operatorname{Ker} \mathscr{B}$, т.е. $(\operatorname{Im} \mathscr{B})^{\perp} \subset \operatorname{Ker} \mathscr{B}$.

2) Обратно, если $X \in \operatorname{Ker} \mathscr{B}$, то $\mathscr{B}(X)=0$. Пусть $Y \in \operatorname{Im} \mathscr{B}$. Тогда существует $Z \in \mathscr{X}(M)$ такое, что $Y=\mathscr{B}(Z)$. Значит, $\langle X, Y\rangle=\langle X, \mathscr{B}(Z)\rangle=-\langle\mathscr{B}(X), Z\rangle$. Итак, $\langle X, Y\rangle=0$. Но $Y \in \operatorname{Im} \mathscr{B}$, откуда $X \in(\operatorname{Im} \mathscr{B})^{\perp}$. Для всех $X \in \operatorname{Ker} \mathscr{B}$ имеем $X \in(\operatorname{Im} \mathscr{B})^{\perp}$, т.е. $\operatorname{Ker} \mathscr{B} \subset \operatorname{Im} \mathscr{B}$. Окончательно получаем, что $(\operatorname{Im} \mathscr{B})^{\perp}=\operatorname{Ker} \mathscr{B}$. Kроме того, если $X \in \operatorname{Ker} \mathscr{B}, Y \in \operatorname{Im} \mathscr{B}$, то $\langle X, Y\rangle=\langle X, \mathscr{B}(Z)\rangle=-\langle\mathscr{B}(X), Z\rangle=0$, т.е. $\operatorname{Ker} \mathscr{B} \perp \operatorname{Im} \mathscr{B}$. Значит, $\mathscr{X}(M)=\operatorname{Ker} \mathscr{B} \oplus \operatorname{Im} \mathscr{B}$.

Tеорема 1. Тензор $\mathscr{B}$ СNK-многообразия класса $R_{1}$ параллелен в связности $\widetilde{\nabla}=\nabla+T$, определяемой формами связности $\widetilde{\omega}=\left\{\widetilde{\omega}_{j}^{i}\right\}, \widetilde{\omega}_{j}^{i}=\omega_{j}^{i}(i, j \neq 0) ; n p u$ этом $\widetilde{\omega}_{o}^{a}=\omega_{o}^{a}+B_{h}{ }^{a} \omega^{h}, \widetilde{\omega}_{a}^{o}=\omega_{o}^{a}+B_{a}{ }^{h} \omega_{h}$.

ДокАзАтЕльство. Так как $\mathscr{B}$ - тензор типа $(1,1)$, имеем

$$
d \mathscr{B}_{j}^{i}+\mathscr{B}_{k}^{i} \widetilde{\omega}_{j}^{k}-\mathscr{B}_{j}^{r} \widetilde{\omega}_{r}^{i}=\mathscr{B}_{j \mid k}^{i} \omega^{k}
$$

где $\left\{\mathscr{B}_{j \mid k}^{i}\right\}$ - компоненты $\widetilde{\nabla} \mathscr{B}$. В частности,

$$
d \mathscr{B}_{b}^{a}+\mathscr{B}_{c}^{a} \widetilde{\omega}_{b}^{c}+\mathscr{B}_{\widehat{c}}^{a} \widetilde{\omega}_{b}^{\widehat{c}}+\mathscr{B}_{o}^{a} \widetilde{\omega}_{b}^{o}-\mathscr{B}_{b}^{c} \widetilde{\omega}_{c}^{a}-\mathscr{B}_{b}^{\widehat{c}} \widetilde{\omega}_{\bar{c}}^{a}-\mathscr{B}_{b}^{o} \widetilde{\omega}_{o}^{a}=\mathscr{B}_{b \mid k}^{a} \omega^{k}
$$

Учитьвая значения компонент оператора $\mathscr{B}(7)$ и (3), получим

$$
\mathscr{B}_{b \mid k}^{a} \omega^{k}=\left(C_{b c}^{a}+B_{[b]}^{h} B_{c] h}^{a}\right) \omega^{c}+\left(C_{b}^{a c}-B_{h}^{[a} B^{c] h} b\right) \omega_{c} .
$$

Так как CNK-многообразие $M$ принадлежит классу $R_{1}$, выражения в скобках обращаются в 0, следовательно, $\mathscr{B}_{b \mid k}^{a} \omega^{k}=0$, а это равносильно равенству $\mathscr{B}_{b \mid k}^{a}=0$. Вычисляя аналогично остальные компоненты тензора $\widetilde{\nabla} \mathscr{B}$, находим $\mathscr{B}_{j \mid k}^{a}=0(i, j, k=1, \ldots, 2 n+1)$, $\widetilde{\nabla} \mathscr{B}=0$.

СлЕдСтвиЕ. Если $M$ - CNK-многообразие класса $R_{1}$, mo $\operatorname{Im} \mathscr{B} u \operatorname{Ker} \mathscr{B}-$ pacпределения определенной размерности. 
ОПРеДЕЛЕнИЕ 2. Рангом CNK-структуры класса $R_{1}$ называется размерность распределения $\operatorname{Im} \mathscr{B}$, т.е. число $\mathrm{rk} \mathscr{B}$. Если $\mathrm{rk} \mathscr{B}=\operatorname{dim} M-1$, то CNK-многообразие $M$ называется CNK-многообразием максимального ранга.

Рассмотрим теперь специализированную $G$-структуру на многообразии $M$ класса $R_{1}$, состоящую из $A$-реперов, первые $r$ векторов $\left\{E_{\alpha}\right\}$ которых лежат в $\operatorname{Im} \mathscr{B}_{p} \oplus \mathbb{C}$, а остальные $\left\{E_{\varphi}\right\}-$ в $\operatorname{Ker} \mathscr{B}_{p} \oplus \mathbb{C}$. Структурные уравнения многообразия $M$ на пространстве этой $G$-структуры запишутся в виде

$$
\begin{aligned}
d \omega= & 2 B_{\alpha}{ }^{\beta} \omega^{\alpha} \wedge \omega_{\beta}, \\
d \omega^{\alpha}= & \theta_{\beta}^{\alpha} \wedge \omega^{\beta}+\theta_{\varphi}^{\alpha} \wedge \omega^{\varphi}+B^{\alpha \beta}{ }_{\gamma} \omega^{\gamma} \wedge \omega_{\beta}+B^{\alpha \beta}{ }_{\varphi} \omega^{\varphi} \wedge \omega_{\beta} \\
& +B^{\alpha \psi}{ }_{\gamma} \omega^{\gamma} \wedge \omega_{\psi}+B^{\alpha \psi}{ }_{\varphi} \omega^{\varphi} \wedge \omega_{\psi}, \\
d \omega_{\alpha}= & -\theta_{\alpha}^{\beta} \wedge \omega_{\beta}-\theta_{\alpha}^{\varphi} \wedge \omega_{\varphi}-B_{\alpha \beta}{ }^{\gamma} \omega_{\gamma} \wedge \omega^{\beta}-B_{\alpha \beta}{ }^{\varphi} \omega_{\varphi} \wedge \omega^{\beta} \\
& -B_{\alpha \psi}{ }^{\gamma} \omega_{\gamma} \wedge \omega^{\psi}-B_{\alpha \psi}{ }^{\varphi} \omega_{\varphi} \wedge \omega^{\psi}, \\
d \omega^{\varphi}= & \theta_{\beta}^{\varphi} \wedge \omega^{\beta}+\theta_{\psi}^{\varphi} \wedge \omega^{\psi}+B^{\varphi \beta}{ }_{\gamma} \omega^{\gamma} \wedge \omega_{\beta}+B^{\varphi \beta}{ }_{\psi} \omega^{\psi} \wedge \omega_{\beta} \\
& +B^{\varphi \psi}{ }_{\gamma} \omega^{\gamma} \wedge \omega_{\psi}+B^{\varphi \psi}{ }_{\xi} \omega^{\xi} \wedge \omega_{\psi}, \\
d \omega_{\varphi}= & -\theta_{\varphi}^{\beta} \wedge \omega_{\beta}-\theta_{\varphi}^{\psi} \wedge \omega_{\psi}-B_{\varphi \beta}{ }^{\gamma} \omega_{\gamma} \wedge \omega^{\beta}-B_{\varphi \beta}{ }^{\psi} \omega_{\psi} \wedge \omega^{\beta} \\
& -B_{\varphi \psi}{ }^{\gamma} \omega_{\gamma} \wedge \omega^{\psi}-B_{\varphi \psi}{ }^{\xi} \omega_{\xi} \wedge \omega^{\psi},
\end{aligned}
$$

где

$$
\theta_{\beta}^{\alpha}=\omega_{\beta}^{\alpha}+B_{\beta}^{\alpha} \omega, \quad \alpha, \beta, \gamma=1, \ldots, r, \quad \varphi, \psi, \xi=r+1, \ldots, 2 n+1 .
$$

На пространстве специализированной $G$-структуры имеем

$$
B_{\alpha}^{\gamma}=0, B_{\varphi}^{\alpha}=0, B_{\varphi}^{\psi}=0
$$

Продифференцируем эти соотношения внешним образом, учитывая выражение (3) для $d B_{\alpha}{ }^{\beta}$ и принадлежность многообразия $M$ классу $R_{1}$ :

$$
\theta_{\beta}^{\varphi}=0, \quad \theta_{\varphi}^{\beta}=0
$$

С учетом (12) система уравнений (11) примет вид

$$
\begin{aligned}
d \omega & =2 B_{\alpha}{ }^{\beta} \omega^{\alpha} \wedge \omega_{\beta}, \\
d \omega^{\alpha} & =\theta_{\beta}^{\alpha} \wedge \omega^{\beta}+B^{\alpha \beta}{ }_{\gamma} \omega^{\gamma} \wedge \omega_{\beta}+B^{\alpha \beta}{ }_{\varphi} \omega^{\varphi} \wedge \omega_{\beta}+B^{\alpha \psi}{ }_{\gamma} \omega^{\gamma} \wedge \omega_{\psi}+B^{\alpha \psi}{ }_{\varphi} \omega^{\varphi} \wedge \omega_{\psi}, \\
d \omega_{\alpha} & =-\theta_{\alpha}^{\beta} \wedge \omega_{\beta}-B_{\alpha \beta}{ }^{\gamma} \omega_{\gamma} \wedge \omega^{\beta}-B_{\alpha \beta}{ }^{\varphi} \omega_{\varphi} \wedge \omega^{\beta}-B_{\alpha \psi}{ }^{\gamma} \omega_{\gamma} \wedge \omega^{\psi}-B_{\alpha \psi}{ }^{\varphi} \omega_{\varphi} \wedge \omega^{\psi}, \\
d \omega^{\varphi} & =\theta_{\psi}^{\varphi} \wedge \omega^{\psi}+B^{\varphi \beta}{ }_{\gamma} \omega^{\gamma} \wedge \omega_{\beta}+B^{\varphi \beta}{ }_{\psi} \omega^{\psi} \wedge \omega_{\beta}+B^{\varphi \psi}{ }_{\gamma} \omega^{\gamma} \wedge \omega_{\psi}+B^{\varphi \psi}{ }_{\xi} \omega^{\xi} \wedge \omega_{\psi}, \\
d \omega_{\varphi} & =\theta_{\varphi}^{\psi} \wedge \omega_{\psi}-B_{\varphi \beta}{ }^{\gamma} \omega_{\gamma} \wedge \omega^{\beta}-B_{\varphi \beta}{ }^{\psi} \omega_{\psi} \wedge \omega^{\beta}-B_{\varphi \psi}{ }^{\gamma} \omega_{\gamma} \wedge \omega^{\psi}-B_{\varphi \psi}{ }^{\xi} \omega_{\xi} \wedge \omega^{\psi} .
\end{aligned}
$$


ОПРЕДЕЛЕНИЕ 3. СNК-многообразие $M$ назовем локально конформным почти контактному метрическому многообразию с замкнутой фундаментальной 2-формой $\Omega$, или Сс-многообразием, если для всех $p \in M$ существует $\sigma \in C^{\infty}\left(U_{p}\right)$ такое, что $\left\{U_{p},\left.e^{-\sigma} \xi\right|_{U_{p}},\left.e^{\sigma} \eta\right|_{U_{p}},\left.e^{2 \sigma} g\right|_{U_{p}}\right\}$ - почти контактное метрическое многообразие с замкнутой фундаментальной 2-формой $\Omega$.

Этот класс многообразий является контактным аналогом локально конформно-келеровых многообразий, широко изучаемых в литературе [12]-[15]. Как и в случае локально конформно-келеровых многообразий, легко показать, что $M$ является Сс-многообразием тогда и только тогда, когда $d \Omega=\Theta \wedge \Omega, d \Theta=0, \boldsymbol{\Theta}(\xi)=0$, где $\boldsymbol{\Theta}=\frac{1}{n-1} \delta \Omega \circ \Phi(\delta-$ оператор кодифференцирования), т.е. $\Theta$ является контактньм аналогом форм Ли. При этом выражения для тензоров $B_{c}^{a b}$ и $B_{a b}{ }^{c}$ имеют вид

$$
B^{a b}{ }_{c}=\alpha^{[a} \delta_{c}^{b]}, \quad B_{a b}{ }^{c}=\alpha_{[b} \delta_{a]}^{c},
$$

где $\left\{\alpha^{a}, \alpha_{a}, \alpha_{o}\right\}$ - компоненты формы Ли $\Theta$ в кобазисе $\left\{\omega^{i}\right\}$.

Пусть распределение $\operatorname{Im} \mathscr{B}$ инволютивно. Тогда оно вполне интегрируемо, и в силу (13) вьполняются следующие условия:

$$
B_{\varphi}^{\alpha \beta}=0, \quad B_{\gamma}^{\alpha \psi}=0, \quad B_{\gamma}^{\varphi \psi}=0, \quad B_{\varphi}^{\alpha \psi}=0
$$

Пусть теперь СNK-многообразие является Сс-многообразием. Из (14) в силу строения тензоров $B^{a b}$ и $B_{a b}{ }^{c}$ находим

$$
\alpha^{\psi}=\alpha^{\beta}=0, \quad \alpha_{\psi}=\alpha_{\beta}=0
$$

Учитьвая соотношения (15), получаем

$$
B_{\gamma}^{\alpha \beta}=B_{\alpha \beta}^{\gamma}=B_{\xi}^{\varphi \psi}=B_{\varphi \psi}^{\xi}=0
$$

Тогда система уравнений (13) примет вид

$d \omega=2 B_{\alpha}{ }^{\beta} \omega^{\alpha} \wedge \omega_{\beta}, \quad d \omega^{\alpha}=\theta_{\beta}^{\alpha} \wedge \omega^{\beta}, \quad d \omega_{\alpha}=-\theta_{\alpha}^{\beta} \wedge \omega_{\beta}, \quad d \omega^{\varphi}=\theta_{\psi}^{\varphi} \wedge \omega^{\psi}, \quad d \omega_{\varphi}=\theta_{\varphi}^{\psi} \wedge \omega_{\psi}$.

Из соотношений (16) видно, что структурные уравнения многообразия $M$ распадаются на две группы: первые три уравнения представляют собой структурные уравнения квазисасакиева многообразия максимального ранга, а последние два - структурные уравнения келерова многообразия [12], [13]. Отсюда теми же рассуждениями, что и в работе [16], получаем, что многообразие $M$ локально эквивалентно произведению квазисасакиева многообразия максимального ранга и келерова многообразия.

Очевидно, что верно и обратное: если многообразие $M$ имеет такую структуру, то его распределение $\operatorname{Im} \mathscr{B}$ инволютивно. Сформулируем полученньй результат в виде теоремы. 
Теорема 2. Пусть $M$ - Сс-многообразие класса $R_{1}$. Распределение $\operatorname{Im} \mathscr{B}$ на многообразии $M$ инволютивно тогда и только тогда, когда многообразие $M$ локально әквивалентно произведению квазисасакиева многообразия максимального ранга и келерова многообразия. В частности, такое многообразие является квазисасакиевым.

Далее, пусть $M-\mathrm{CNK}$-многообразие. Сравним значения компонент $R^{a}$ obo тензора Римана-Кристоффеля и $\mathscr{B}_{o, b}^{a}$ оператора $\nabla \mathscr{B}$, заданные формулами (6) и (8): $R^{a}{ }_{\text {obo }}=$ $-\mathscr{B}_{o, b}^{a}$. Кроме того, имеем $R^{\widehat{a}}{ }_{\text {obo }}=R^{o}{ }_{\text {obo }}=0, \mathscr{B}_{o, b}^{\widehat{a}}=\mathscr{B}_{o, b}^{o}=0$. Но из $R^{a}{ }_{o b o}=-\mathscr{B}_{o, b}^{a}$ следует, что

$$
\left(R\left(E_{b}, E_{o}\right) E_{o}\right)^{a}=-\left(\nabla_{E_{b}}(\mathscr{B}) E_{o}\right)^{a}
$$

а также

$$
\left(R\left(E_{b}, E_{o}\right) E_{o}\right)^{\widehat{a}}=\left(\nabla_{E_{b}}(\mathscr{B}) E_{o}\right)^{\widehat{a}}, \quad\left(R\left(E_{b}, E_{o}\right) E_{o}\right)^{o}=\left(\nabla_{E_{b}}(\mathscr{B}) E_{o}\right)^{o} .
$$

Из этих тождеств имеем $R\left(E_{b}, E_{o}\right) E_{o}=-\nabla_{E_{b}}(\mathscr{B}) E_{o}$. Рассуждая как и вьше, получаем

$$
R(X, \xi) \xi=-\nabla_{X}(\mathscr{B}) \xi, \quad X \in \mathscr{X}(M) .
$$

Назовем последнее тождество вторым тождеством кривизны CNK-многообразия.

ОПРЕДЕЛЕНИЕ 4. СNК-многообразие $M$ назьвается многообразием класса $R_{2}$, если $R(X, \xi) \xi=0, X \in \mathscr{X}(M)$.

Из (17) следует, что CNK-многообразие принадлежит классу $R_{2}$ тогда и только тогда, когда $\nabla_{X}(\mathscr{B}) \xi=0, X \in \mathscr{X}(M)$. Из соотношений $(7),(8)$ получаем, что если $\mathrm{CNK}$-многообразие является многообразием класса $R_{2}$, то $\mathscr{B}^{2}=0$. С другой стороны, по предложению 1 вьполняется тождество

$$
\langle\mathscr{B}(X), Y\rangle=-\langle X, \mathscr{B}(Y)\rangle, \quad X, Y \in \mathscr{X}(M) .
$$

Поэтому $\langle\mathscr{B}(X), \mathscr{B}(Y)\rangle=-\left\langle X, \mathscr{B}^{2}(Y)\right\rangle=0$, т.е. $\langle\mathscr{B}(X), \mathscr{B}(Y)\rangle=0, X, Y \in \mathscr{X}(M)$. В частности, $\langle\mathscr{B}(X), \mathscr{B}(X)\rangle=0$, т.е. $\|\mathscr{B}(X)\|=0, X \in \mathscr{X}(M)$, значит, и $\mathscr{B}(X)=0$, $X \in \mathscr{X}(M)$. Следовательно, $\mathscr{B}=0$. Обратное очевидно. Следовательно, доказана

Теорема 3. CNK-многообразие $M$ принадлежст классу $R_{2}$ тогда и только тогда, когда $\mathscr{B}=0$.

Пусть CNK-многообразие $M$ принадлежит классу $R_{2}$. Тогда $B_{a}{ }^{b}=0$. Легко показать, что для CNK-многообразия тензор Нейенхейса будет иметь вид

$$
N(X, Y)=-\frac{1}{2}\langle\mathscr{B}(X), Y\rangle \xi, \quad X, Y \in \mathscr{X}(M) .
$$

В силу того, что многообразие $M$ принадлежит классу $R_{2}$, получаем $N(X, Y)=0$, $X, Y \in \mathscr{X}(M)$. Это условие означает интегрируемость почти контактной метрической структуры [6]. Принимая во внимание обозначение (2) для $B_{a}{ }^{b}$ и принадлежность CNK-многообразия классу $R_{2}$, будем иметь $\eta\left(\nabla_{E_{\widehat{b}}}(\Phi) E_{a}\right)=0$. Так как $E_{a}, E_{\widehat{b}}-$ базисные векторы пространств $D_{\Phi}^{\sqrt{-1}}$ и $D_{\Phi}^{-\sqrt{-1}}$ соответственно, имеем

$$
\eta\left(\nabla_{X}(\Phi) Y\right)=0, \quad X \in D_{\Phi}^{-\sqrt{-1}}, \quad Y \in D_{\Phi}^{\sqrt{-1}} .
$$


Из этого равенства следует, что

$$
\eta\left(\nabla_{\bar{\sigma} \tilde{X}}(\Phi)(\sigma \widetilde{Y})\right)=0
$$

где $\tilde{X}, \tilde{Y} \in \mathscr{L}^{\mathbb{C}}$, а $\sigma=\frac{1}{2}(\mathrm{id}-\sqrt{-1} \Phi), \bar{\sigma}=\frac{1}{2}(\mathrm{id}+\sqrt{-1} \Phi)$ - проекторы на распределения $D_{\Phi}^{\sqrt{-1}}$ и $D_{\Phi}^{-\sqrt{-1}}$ соответственно. Раскроем это выражение по линейности

$$
\eta\left(\nabla_{\tilde{X}}(\Phi) \tilde{Y}+\nabla_{\Phi \tilde{X}}(\Phi)(\Phi \tilde{Y})-\sqrt{-1} \nabla_{\tilde{X}}(\Phi)(\Phi \tilde{Y})+\sqrt{-1} \nabla_{\Phi \tilde{X}}(\Phi) \tilde{Y}\right)=0 .
$$

Как и выше, это тож дество эквивалентно обращению в 0 его вещественной части, т.е.

$$
\eta\left(\nabla_{\tilde{X}}(\Phi) \tilde{Y}+\nabla_{\Phi \tilde{X}}(\Phi)(\Phi \tilde{Y})\right)=0 .
$$

Учитьвая, что $\mathscr{L}=\operatorname{Im} \Phi$, имеем

$$
\eta\left(\nabla_{\Phi X}(\Phi) \Phi Y+\nabla_{\Phi^{2} X}(\Phi)\left(\Phi^{2} Y\right)\right)=0, \quad X, Y \in \mathscr{X}(M) .
$$

Кроме того, компонента $\Phi_{\widehat{a}, \widehat{b}}^{o}$ тензора $\nabla \Phi \mathrm{CNK-многообразия} \mathrm{имеет} \mathrm{значение} \Phi_{\widehat{a}, \widehat{b}}^{o}=0$. Проводя аналогичные рассуждения, получаем

$$
\eta\left(\nabla_{\Phi X}(\Phi) \Phi Y-\nabla_{\Phi^{2} X}(\Phi)\left(\Phi^{2} Y\right)\right)=0, \quad X, Y \in \mathscr{X}(M) .
$$

Очевидно, что выражения (18), (19) эквивалентны тождеству

$$
\eta\left(\nabla_{\Phi X}(\Phi) \Phi Y\right)=0, \quad X, Y \in \mathscr{X}(M) .
$$

Пусть теперь СNK-структура интегрируема. Рассмотрим системы Пфаффа: $\omega=0$ и $\omega^{a}=\omega_{a}=0, a=1, \ldots, n$. Эти системы вполне интегрируемы в силу (1). Значит, через каждую точку многообразия $M$ проходит интегральное многообразие максимальной размерности каждой из систем. Ясно, что интегральное многообразие, определяемое системой Пфаффа $\omega=0$, имеет размерность $2 n$, а интегральное многообразие системы $\omega^{a}=\omega_{a}=0$ имеет размерность 1 .

Далее, из соотношений (1) непосредственно следует, что вторая квадратичная форма интегральных многообразий каждого из распределений - нулевая, и следовательно, эти интегральные многообразия являются вполне геодезическими.

Кроме того, распределения, определяемые системами Пфаффа $\omega=0$ и $\omega^{a}=\omega_{a}=0$, взаимно-дополнительные. В самом деле, система Пфаффа $\omega=0$ определяет распределение $\mathscr{L}=\operatorname{Ker} \eta$, а система Пфаффа $\omega^{a}=\omega_{a}=0$ определяет распределение $\mathscr{M}=\operatorname{Ker} d \eta$. Очевидно, $\mathscr{L} \cap \mathscr{M}=\{0\}, \mathscr{L} \perp \mathscr{M}, \mathscr{X}(M)=\mathscr{L} \oplus \mathscr{M}$. При этом первая групша структурных уравнений CNK-многообразия имеет вид

$$
d \omega=0, \quad d \omega^{a}=\omega_{b}^{a} \wedge \omega^{b}+B^{a b}{ }_{c} \omega^{c} \wedge \omega_{b}, \quad d \omega_{a}=-\omega_{a}^{b} \wedge \omega_{b}+B_{a b}{ }^{c} \omega^{b} \wedge \omega_{c} .
$$

Распределения $\mathscr{L}$ и $\mathscr{M}$ инвариантны относительно оператора $\Phi$. Значит, можно рассмотреть сужение оператора $\Phi$ на эти распределения. Сужение $\left.\Phi\right|_{\mathscr{L} \mathbb{C}}$ определяет почти комплексную структуру на интегральных многообразиях этого распределения. Последние две грушшы уравнений (20) являются структурными уравнениями эрмитова многообразия. С учетом сделанных замечаний так же, как и в работе [16], получаем, что многообразие $M$ локально эквивалентно произведению эрмитова многообразия на вещественную прямую. Здесь под локальной эквивалентностью, как и в [16], понимается диффеоморфизм, сохраняющий дополнительную структуру многообразия (в данном случае, почти контактную метрическую структуру). Сформулируем полученньй результат в виде теоремы. 
ТеоремА 4. Для CNK-многообразия $M$ әквивалентны следующие утверэсдения:

1) $M$ принадлежит классу $R_{2}$;

2) справедливо тождество $\eta\left(\nabla_{\Phi X}(\Phi) \Phi Y\right)=0, X, Y \in \mathscr{X}(M)$;

3) $М$ имеет интегрируемую структуру;

4) $M$ локально әквивалентно произведению әрмитова многообразия на вещественную прямую.

В заключение автор выражает глубокую благодарность профессору В. Ф. Кириченко за постановку проблемы, внимание и помощь, оказанные в ходе работе наднастоящей статьей.

\section{СПИСОК ЦИТИРОВАННОЙ ЛИТЕРАТУРЫ}

[1] Sasaki S., Hatakeyma J. On differentiable manifolds with contact metric structures // J. Math. Soc. Japan. 1964. V. 14. P. 249-271.

[2] Ishihara I. Anti-invariant submanifolds of a Sasakian space form // Kodai Math. J. 1976. V. 2. P. $171-186$.

[3] Tanno S. Qusi-Sasakian structures of rank $2 p+1 / /$ J. Differential Geom. 1971. V. 5. № 3-4. P. 317-324.

[4] Ianus S., Visinescu M. Kaluza-Klein theory with scalar fields on generalised Hopf manifolds // Classical Quantum Gravity. 1987. V. 4. № 5. P. 1317-1325.

[5] Gray A. Curvature identites for Hermitian and almost Hermitian manifolds // Tôhoku Math. J. 1976. V. 28. № 4. P. 601-612.

[6] Goldberg S., Yano K. Integrability of almost cosymplectic structures // Pacific J. Math. 1969. V. 31. № 2. P. 373-382.

[7] Кириченко В.Ф. Методы обобщенной эрмитовой геометрии в теории почти контактных многообразий // Итоги науки и техн. Пробл. геометрии. Т. 18. М.: ВИНИТИ, 1986. С. 25-71.

[8] Ефимов Н. В., Розендорн Э. Р. Линейная алгебра и многомерная геометрия. М.: Наука, 1970.

[9] Кириченко В.Ф. Аксиома Ф-голоморфных плоскостей в контактной метрической геометрии // Изв. АН СССР. Сер. матем. 1983. Т. 48. № 4. С. 711-734.

[10] Кобаяси Ш., Номидзу К. Основы дифференциальной геометрии. Т. 1, 2. М.: Наука, 1981.

[11] Картан Э. Риманова геометрия в ортогональом репере. М.: Изд-во МГУ, 1960.

[12] Кириченко В.Ф. Локально конформно-келеровы многообразия постоянной секционной кривизны // Матем. сб. 1991. Т. 182. № 3. С. 354-363.

[13] Кириченко В.Ф. Конформно-плоские локально конформно-келеровы многообразия // Матем. заметки. 1992. Т. 51. № 5. С. 57-66.

[14] Vaisman I. On locally conformal Kähler manifolds // Israel J. Math. 1976. V. 24. № 3-4. P. 338-351.

[15] Vaisman I. On locally and globally conformal Kähler manifolds // Trans. Amer. Math. Soc. 1980. V. 2. P. 533-542.

[16] Кириченко В. Ф. Квазиоднородные многообразия и обобщенные почти эрмитовы структуры // Изв. АН СССР. Сер. матем. 1983. Т. 47. №6. С. 1208-1223. 